\title{
Singularities in Qualitative Reasoning ${ }^{\star}$
}

\author{
Björn Gottfried \\ Centre for Computing Technologies \\ University of Bremen, Germany \\ bg@tzi.de
}

\begin{abstract}
Qualitative Reasoning is characterised by making knowledge explicit in order to arrive at efficient reasoning techniques. It contrasts with often intractable quantitative models. Whereas quantitative models require computations on continuous spaces, qualitative models work on discrete spaces. A problem arises in discrete spaces concerning transitions between neighbouring qualitative concepts. A given arrangement of objects may comprise relations which correspond to such transitions, e.g. an object may be neither left of nor right of another object but precisely aligned with it. Such singularities are sometimes undesirable and influence underlying reasoning mechanisms. We shall show how to deal with singular relations in a way that is more closely related to commonsense reasoning than treating singularities as basic qualitative concepts.
\end{abstract}

\section{Introduction}

In this essay we shall discuss problems arising by describing arrangements of objects qualitatively. We are concerned with the relations depicted in Fig. 1, which have been introduced in [3]. We refer to the set of these relations as $\mathcal{B A}$. The relations in $\mathcal{B A}$ describe arrangements of intervals in the two-dimensional plane qualitatively; they can be considered as the two-dimensional analogue of Allen's one-dimensional interval relations [1]. $\mathcal{B A}$ is distinguished from other qualitative representations (cf. [2]) in that it comprises only disconnection relations. Relations between disconnected objects are of interest in a number of areas, mainly when spatiotemporal interactions between objects are to be described. It could be argued that connection relations are equally important. But there are no connections, for example, between road-users in traffic, pedestrians walking in a market square, sportsmen playing on a pitch, or generally between objects forming patterns of spatiotemporal interactions. Sometimes the distances between objects become very small, but they still remain detached from one another and can generally change their orientation and position independently of other objects. We are simply interested in possible relations between objects that are not connected.

\footnotetext{
* Appeared in: B. Gottfried, "Singularities in Qualitative Reasoning", In B. Buchberger \& J. Campbell (Eds.), AISC 2004 7th Int. Conf. on Artificial Intelligence and Symbolic Computation (p. 276-280), Sept. 2004, Castle of Hagenberg, Linz, Austria: Springer-Verlag.
} 
It is less a question of motivating the necessity of different disconnection relations, than of restricting the relations to those in general positions. In $\mathcal{B A}$ the endpoints of all intervals are in general positions. The examples on the right of Fig. 1 show relations in singular positions. These correspond to special cases in which intervals are precisely aligned with each other. But in $\mathcal{B A}$ there are no such singular relations explicitly defined. For instance, there is no relation between $F_{l}$ and $F O_{l}$ which would correspond to an interval in which one endpoint is located exactly level with an endpoint of the other interval (see Fig. 1.(a)). The question arises as to how we deal with such singular arrangements, in which one endpoint lies precisely at a location which marks the transition between qualitative concepts, as between $F_{l}$ and $F O_{l}$. This is important, since no possible arrangement of intervals should remain undefined. This is the issue we are interested in.

\section{Singular Relations}

First of all we show why singular relations exist at all. A qualitative representation is the result of an abstraction process, which can be regarded as the partitioning of a continuous space into a number of equivalence classes. As a sideeffect of this, singularities emerge as transitions between neighbouring classes. For example, the continuous space of interval arrangements in $\mathbb{R}^{2}$ can be conceived as consisting of all metrically distinct interval arrangements. A special abstraction of this continuous space distinguishes the relations of $\mathcal{B A}$, in which each equivalence class is a binary relation between two intervals. In the neighbourhood graph in Fig. 1, the transition between two neighbouring classes marks a singularity, for instance, between $F_{l}$ and $F O_{l}$. We refer to the interval relations which fall into these transitions as singular relations. Fig. 1.(a) serves as an example. An arbitrary small change in the position of one interval which forms a singular relation with another interval transforms it in most cases into a general relation. Such a small change applied to a general relation would not normally change this relation.

We would like to argue that singular relations should not have the status of basic relations in a qualitative representation. By contrast to $\mathcal{B} \mathcal{A}$, which comprises only 23 relations, there exist 226 relations when we additionally consider singular relations [3] - a significant difference since all these relations need to be distinguished when analysing and interpreting situations. More importantly, singular relations are somewhat misplaced in the context of qualitative reasoning. We are not at all interested in whether objects are precisely aligned. We focus on coarse relations between objects, which are simple to obtain and which allow efficient commonsense reasoning. For instance, we want to know whether one object is to the left of another one, whether it is moving in the same direction, and the like. What distinguishes qualitative relations from metrical relations is that they can be recognised easily by perception. However, this does not apply to singular relations, which require precise measurements. We conclude that singular relations are not compatible with commonsense reasoning, although there 


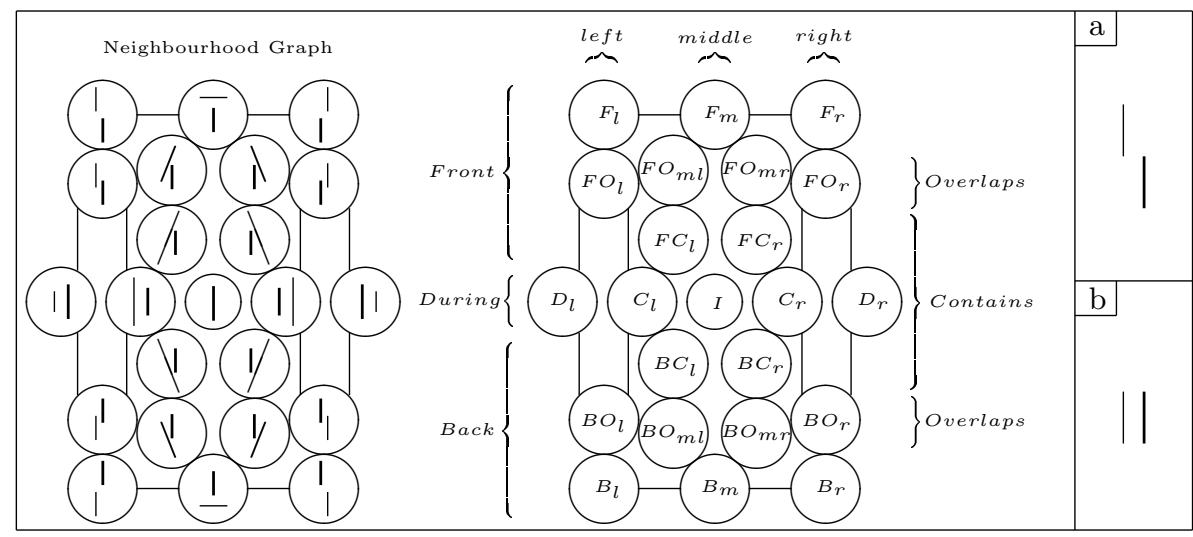

Fig. 1. Left: Interval relations embedded in two dimensions; the vertical reference interval is displayed bold. Middle: A mnemonic description; Right: Singular relations

are exceptions in those fields where singular relations are as easy to obtain as general relations. For example, in the case of events we often know whether one event follows another one directly: after the performance a reception is held in the foyer; there is no time to go shopping between the performance and the reception - these events meet in time.

\subsection{Representing Singular Relations}

Having said that singular relations are incompatible with the idea of commonsense reasoning - more so in two dimensions than in one - we must show how to deal appropriately with singular relations. We cannot simply exclude them, since we need to represent every conceivable arrangement of intervals. One way of dealing with them consists in assigning singular relations to similar general relations. The singular relation in the first example could be assigned to $F_{l}$, since there is only one point that is not actually in relation $F_{l}$; this may be an appropriate solution in applications in which coarse reasoning is performed. But when such a precise distinction matters we are outside the scope of qualitative reasoning.

The second example, Fig. 1.(b), is more difficult to handle. If we regard this arrangement as $D_{l}$ then we are heading for a problem. What about the converse relation? If it is regarded as $D_{r}$, then it holds for both intervals that each is contained in the other one - a quite awkward situation. For this reason we have to proceed as we do whenever we encounter indeterminate information in any qualitative representation: by sets of possible relations. In Fig. 1.(b) we would represent the singular relation by $\left\{D_{l}, C_{l}, F O_{l}, B O_{l}\right\}$ and its converse by $\left\{D_{r}, C_{r}, F O_{r}, B O_{r}\right\}$. In this way, we can deal with parallel intervals which are equal in length. The representation does not seem to be very precise, but precision is exactly what we want to avoid in a qualitative representation. When can 
we be sure whether parallel lines really are equal in length? Only when we have precise measuring tools. Isn't there always a little uncertainty left when working without such tools? At most we know that two lines in a given arrangement are likely to be equal in length, but at the same time we also know that they may be something else - something similar. Similar relations form a neighbourhood in the $\mathcal{B A}$-graph, and such neighbourhoods circumscribe the singular relations. Accordingly $\left\{D_{l}, C_{l}, F O_{l}, B O_{l}\right\}$ would seem to be quite an appropriate description of what we really know about two parallel lines which are probably equal in length.

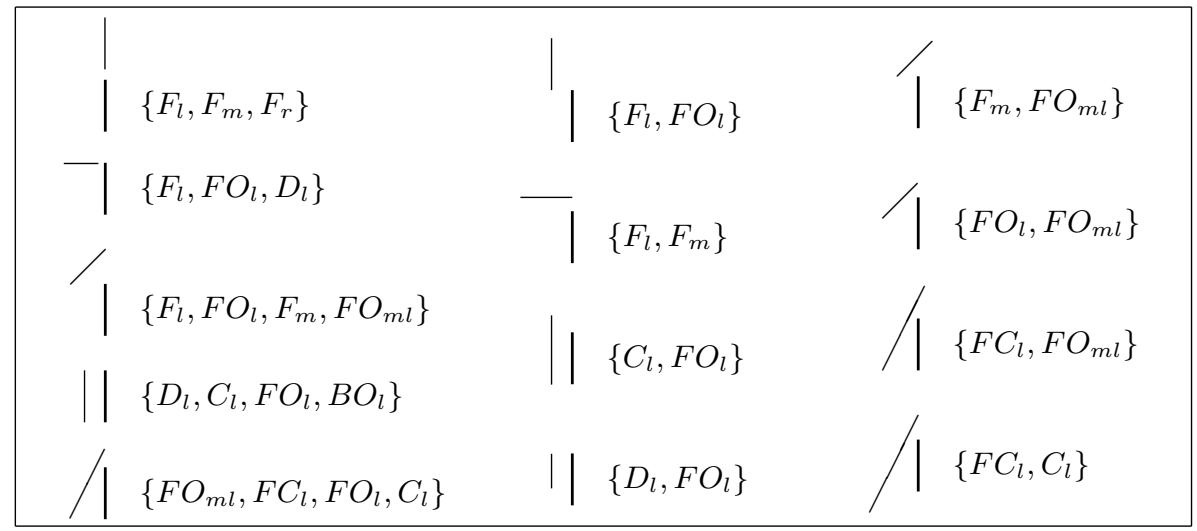

Fig. 2. Singular relations defined by sets of general relations

Fig. 2 shows how singular relations are represented by sets of general relations. Only a quarter of all relations are depicted, since the other relations are symmetrical to those in Fig. 2. As with the disconnection relations of $\mathcal{B A}$, only disconnected singularities are considered. Apparently connected singularities are treated as apparently connected general relations, i.e. they are conceived as disconnected relations in which distances become arbitrarily short. Our knowledge gets more uncertain near singular relations - this uncertainty is represented by sets comprising a number of possible relations rather than only one relation. In particular, if two endpoints are in singular positions then these sets consist of three or four general relations, depending on whether the endpoints lie on the same singularity, e.g. $\left\{F_{l}, F_{m}, F_{r}\right\}$ in Fig. 2, or on different singularities, e.g. $\left\{D_{l}, C_{l}, F O_{l}, B O_{l}\right\}$. By contrast, if there is only one endpoint in singular position the sets consist of only two general relations. We observe that all singularities are uniquely identified by this technique.

\subsection{Reasoning with Singular Relations}

How does this representation of singular relations affects reasoning processes? Let us consider the example in Fig. 3. We assume that we know the relations 


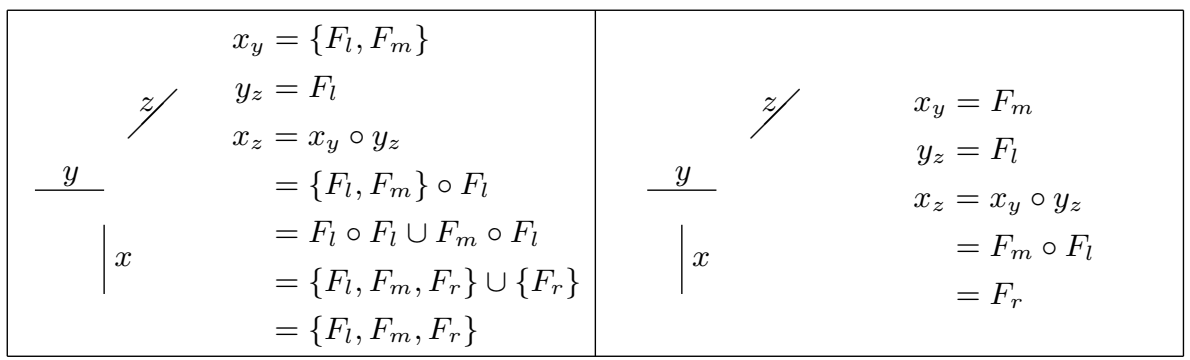

Fig. 3. Transitivity with a singular relation (left), and without any singularity (right)

between $x$ and $y$ as well as those between $y$ and $z$. For the position of $y$ with respect to $x$ we write $x_{y}$, and accordingly we write $y_{z}$ for the position of $z$ with respect to $y$. Our goal is to infer the relationship between $z$ and $x$, i.e. $x_{z}$. We do this by the composition operation which was defined in [3]: for each pair of general relations the transitivity relation is given. The left hand side of Fig. 3 shows $x_{y}$ in singular relation; the composition result is indeterminate. In comparison, the right hand side of Fig. 3 shows $x_{y}$ in general relation; here the composition result is less indeterminate. Note that we assume that $x_{z}$ cannot be perceived directly, as is actually the case in this figure.

\section{Discussion}

Hitherto qualitative representations have treated singular relations as being on a par with general relations. This is useful in some areas, for example, in order to distinguish whether an event happens before another event, or whether it immediately follows (meets) another one [1]. We have argued that singular relations are not as important as general relations in some applications, and that they form a different sort of relation since they do not accord with commonsense reasoning. Characterising singularities on the basis of neighbourhoods, we have treated them as relations of second order rather than basic relations. As a consequence, the endpoints of basic relations always lie in general positions. Indeed $\mathcal{B A}$ forms a set of relations which covers all possible situations when circumscribing singular relations by neighbourhoods of general relations $-\mathcal{B A}$ leaves nothing undefined. This also holds for other qualitative representations.

To summarise, we have identified singularities as artefacts in qualitative representations. They are problematic in some areas, in that they require precise measurements whereas precision is normally avoided in qualitative reasoning. We have outlined how to deal with singularities by means of sets of possible relations, i.e. by defining singularities as sets of general relations.

\section{References}

1. J. F. Allen. Maintaining knowledge about temporal intervals. Communications of the ACM, 26(11):832-843, 1983. 
2. A. G. Cohn and S. M. Hazarika. Qualitative spatial representation and reasoning: An overview. Fundamenta Informaticae, 43:2-32, 2001.

3. B. Gottfried. Reasoning about intervals in two dimensions. In IEEE Int. Conference on Systems, Man and Cybernetics, The Netherlands, 2004. 